\title{
Regulation of the stress response by social buffering: A review across species d
}

\author{
Matías Alejandro Avellaneda 느, \& Giselle Kamenetzky (i) 12 \& 3
}

Universidad de Buenos Aires, Buenos Aires, Argentina.

\begin{abstract}
This review presents a critical reading of the literature on social buffering in human and non-human animals. The term social buffering has been coined to refer to an attenuation of stress responses by the presence of conspecifics. Evidence shows that the buffer seems to be specific for each stage of development, being the mother the factor that attenuates the stress responses during early development and conspecifics of the same age, later in life. An animal model of scarcity of resources revealed that when being reared by a stressed mother, the social buffering effect does not occur. The literature reviewed allows us to approach a key factor related to stress and its effects in the different stages of ontogeny.
\end{abstract}

\section{Keywords}

stress; social buffering; scarcity model; odor; respondent conditioning

\section{RESUMEN}

Esta revisión presenta una lectura crítica de la literatura sobre social buffering en animales humanos y no humanos. El término social buffering ha sido designado para referirse a la atenuación de las respuestas de estrés por la presencia de congeners. La evidencia muestra que el estímulo atenuador es específico para cada fase del desarrollo, siendo la madre el factor que atenúa las respuestas de estrés durante las etapas tempranas, y los congéneres de la misma edad en etapas más tardías. Un modelo animal de escasez de recursos reveló que, bajo crianza por una madre estresada, the efecto de social buffering no ocurre. La literatura revisada permite dilucidar un factor clave relacionado al estrés y a sus efectos en distintas etapas de la ontogenia.

Palabras Claves

estrés; amortiguación social; modelo de escasez; olor; condicionamiento del encuestado.

\footnotetext{
1 Correspondence about this article should be addressed to Matias Alejandro Avellaneda: m.avellaneda@live.com.ar

2 Conflicts of Interest: The authors declare that the research was conducted in the absence of any commercial or financial relationships that could be construed as a potential conflict of interest.

3

Instituto de Investigaciones Médicas Dr. Alfredo Lanari
} 
Regulación de la respuesta al estrés mediante el amortiguamiento social - una revisión entre especies

\section{Introduction}

Stress has been studied in a variety of conditions by different authors, given its relevance in psychological, immune, neurological, endocrinological and social aspects, among others (e.g., Biggs et al., 2017; Levine \& Ursin, 1991; Ursin, 1991. For a historical account of the concept, see Cooper \& Dewe, 2008). One definition commonly used is any "real or interpreted threat to the physiological or psychological integrity of an individual that results in physiological and/or behavioral responses" (McEwen, 2000, p. 508, cited in Hostinar et al., 2014). Physiologically, it is associated with the activity of the hypothalamo-pituitary-adrenocortical (HPA) axis, composed by the hypothalamus, the pituitary gland, the adrenal glands, and the interactions among them (for a detailed description of the anatomy and function of the HPA axis, see Watss, 2007). Briefly, the paraventricular nucleus of the hypothalamus contains neuroendocrine neurons that synthesize corticotropin-releasing hormone $(\mathrm{CRH})$, which regulate the anterior lobe of the pituitary gland. Particularly, CRH stimulates adrenocorticotropic hormone (ACTH) secretion, which in turn stimulates the adrenal cortex to produce glucocorticoid hormones (mainly cortisol in primates and corticosterone in rodents). These hormones suppress CRH and ACTH production in a negative feedback cycle (Herman \& Cullinan, 1997).

Part of the interest in stress research stems from the fact that prolonged HPA axis activation has proven to be detrimental to health. This is called chronic stress (in contrast with acute stress, which occurs in response to an acute threat), and has been shown to produce both structural and functional changes in the nervous system (Duval at al., 2010). For example, chronic stress has been linked to depression (Dinan, 1994; Gold et al., 2015; Redinbaugh et al., 1995), immunological deficiencies (Yada \& Tort, 2016), cardiovascular alterations (Cohen et al., 2015) and inflammatory diseases (LeResche \& Dworkin, 2002). Furthermore, stress has also been proven to produce social (Veenit et al., 2014) and cognitive (McEwen \& Sapolsky, 1995; Pechtel \& Pizzagalli, 2011) alterations. Even though stress responses are generally associated with negative consequences, there is evidence that exposure to stressors in previous phases or in early ontogeny, depending on the nature and intensity of the stressor, predisposes to an increase in resistance to stress in later stages. For example, the effect of partial reinforcement on extinction, exposure to partial reinforcement (which means being exposed to stress by 
omission of a reinforcer on repeated occasions) significantly increase resistance to extinction, which reflects that the persistence of a response undergoing extinction is greater when training consisted of partial reinforcement (not all trials are reinforced after executing a response) as compared to continuous reinforcement (all trials are reinforced; Bouton et al., 2014).

Taking this into consideration, a vast effort has been made to find means to prevent negative effects of stress. For example, it has been well-established that mindfulness meditation (Grossman et al., 2004) and the capacity to control the stressful stimuli (Hanson et al., 1976) can prevent the elevation in glucocorticoids associated with HPA axis activity. Another variable that has consistently been shown to attenuate the negative effects of stress is social support, which can be defined as any informational cue suggesting that one is appreciated/loved by other people, which together form a network of mutual obligations (Cobb, 1976). More generally, social stimuli are capable of exerting a beneficial effect under stressful situations, as we will see in the next section. The goal of this article is to provide a narrative review of the literature on social buffering, by exploring different aspects of this phenomenon that may be of interest.

\section{Method}

We conducted a comprehensive search of the Google Scholar database, without filtering by date. Different searches were conducted during the period ranging from April 2019 to June 2019. An additional search was conducted during February and March 2021 to include articles published during 2019 and 2020. Keywords used included social buffering, resource scarcity, conditioned fear inhibition, and social buffering by olfactory stimuli. Articles obtained were reviewed and included when considered appropriate. These decisions were made by the two authors, who have wide experience conducting research with regard to the influence of social stimuli during the early ontogeny. Existent reviews of the literature were also consulted to find additional articles of interest. Research articles referenced by all or most of these reviews were deemed appropriate and also included in the present review.Only publications in English and fully published were considered for inclusion. The main summary measure in the reviewed literature was the difference between means among the groups assessed. In an attempt to diminish bias risk, articles from different authors and laboratories were included, even when their results differed from those usually found in the literature. 


\section{Results}

A total of 31 articles were considered for inclusion, none of which were excluded. A summary of their main characteristics is included in Table 1 in the Conclusions section

\section{The social buffering effect}

Activity of the HPA axis has been shown to be regulated by a wide range of social stimuli. Specifically, the release of stress-related hormones (cortisol and corticosterone) has proven to be attenuated by the presence of conspecifics. The term social buffering has been coined to refer to this phenomenon (Gunnar \& Hostinar, 2015; Hennesy et al., 2009; Hostinar et al., 2014), which has been theoretically developed for over four decades (Cohen \& McKay, 1984; Cohen \& Wills, 1985; Dean \& Lin, 1977).

This effect has been observed in a wide variety of species, including humans (e.g., Ditzen et al., 2007; Kirschbaum, Klauer, Filipp \& Hellhammer, 1995), squirrel monkeys (e.g., Hennessy, 1984), rats (e.g., Stanton et al., 1987) guinea pigs (e.g., Hennessy et al., 2006), horses (e.g., Ricci-Bonot, 2021), and fish (e.g., Culbert et al., 2019) under different situations such as mild everyday stressors (Albers et al., 2008), maternal separation (Coe et al., 1978) and public speech (Kirschbaum et al., 1993). Neurophysiological correlates of the effect have also been investigated (Einsenberger et al., 2007; Taylor et al., 2008). In this section, some of the most representative experiments regarding this effect are reviewed. All of these works were carried out under situations of acute stress (some works regarding chronic stress are reviewed in a later section). Research with humans, nonhuman primates and rodents are presented separately.

\section{Social buffering in humans}

The ability of different individuals to buffer the stress response has been shown to depend on the stage of development of the subjects. A widely tool used in stress-related human research is the Trier Social Stress Test (TSST; Kirschbaum et al., 1993), which consists of a 10-minute anticipation period followed by a 10-minute test period (during which subjects are instructed to perform a task such as a free speech in front of an audience). The TSST has been shown to produce a significant increase in cortisol levels (Abelson et al., 2014; Childs et al., 2006; Kelly et al., 2008). Parents proved to be successful in reducing the stress response in children under the TSST when compared toa 
stranger, while no difference was found in adolescents (Hostinar et al., 2015).These results suggest that the effectiveness of the buffer depends on the stage of development.

Interestingly, the physical presence of the person providing support is not essential for social buffering to occur. Seltzer, Ziegler, and Pollak (2010) studied the recovery from the TSST in 7-12-year-old girls. Three conditions were considered: (1) recovery, after the task, in the presence of their mother, (2) recovery with an experimenter and the possibility of speaking to their mother, and (3) recovery with the experimenter. Even though condition (1) was found to be the most effective in reducing the cortisol level produced by the task, condition (2) also proved to be effective when compared to condition (3). This effect is not observed, however, when only written mother-child exchanges are available; in this case, children allowed to type and receive instant messages with their mother show no difference in cortisol levels with respect to children with no contact at all (Seltzerv et al., 2012). These results suggest that specific types of stimulation may be necessary for social buffering to occur. In accord with this - as will be discussed in a later section - the evidence in studies with rats show that the odors present in the context of stress appear to be fundamental for social buffering to occur.However, qualitative aspects of the relationship established with the potential buffer have proved to be important in determining whether or not inhibition of the stress response would occur. For example, Albers et al. (2008) found that maternal quality (as measured from videotapes) predicted whether or not the stress response to a mild stressor would be inhibited in infants. Similarly, Nachmias, Gunnar, Mangelsdorf, Parritz, and Buss (1996) found higher cortisol levels in toddlers exposed to novel situations when the attachment with the mother was judged to be insecure rather than secure.

Using the TSST protocol, Kirschbaum et al., (1995) found sex-specific effects of social support in older humans. In this study, adult subjects were assigned to one of three groups; no support, support from a stranger, or support from the boyfriend/girlfriend (partner). In the latter two conditions, supporters were instructed to provide social support during the anticipation period. While partner-supported men showed lower cortisol levels with respect to the other two conditions, the authors found that partner-supported women exhibited higher cortisol levels compared to the stranger condition. These results seem to indicate that women are less susceptible to social buffering by verbal social support than men are. Further evidence of this comes from an experiment carried out by Ditzen et al., (2007). These authors assigned adult women to one of three groups for the anticipation phase; (1) ten minutes of physical interaction with their partner (neck and shoulder 
massage), (2) ten minutes of verbal interaction with their partner or (3) no interaction with their partner. As in the previous study, no difference was found among conditions (2) and (3). However, subjects in group (1) showed lower levels of cortisol relative to the other two, indicating that women can benefit from social support from their partner, as long as physical contact is involved.

Gender-specific effects were also reported by Glynn, Christenfeld, and Gerin (1999). These authors conducted an experiment in which male and female adults were instructed to give a speech, and received either supportive or unsupportive feedback from a male or female observer. Results from this experiment showed that positive feedback from a female observer was effective in reducing stress responses when compared to negative feedback in both men and women. This difference was not observed when the observer was male; in this case both groups of subjects showed similar levels of stress responsiveness. This study also shows that people other than partners can modulate the stress response in humans. Indeed, Heinrichs, Baumgartner, Kirschbaum, and Ehlert (2003) showed that close friends function as sources of social buffering in adult men. In this experiment, participants were instructed either to bring their best friend (support condition) or to go alone (no support condition) to a TSST task. In the former condition, the friends were instructed to be as helpful as possible to the participants during the speech preparation (anticipation period) and to provide instrumental as well as emotional support. As expected, lower cortisol levels were found among subjects in the support condition. Similarly, Adams, Santos, and Bukowski, (2011) found that children undergoing daily situations experienced as negative in the presence of their best friend had a lower cortisol increase when compared to children who were not accompanied by their friend in such circumstances.

This topic was studied with animal models, using several species. The following section presents research that provides evidence about the reduction of cortisol in a stress situation, in the presence of social stimuli.

\section{Social buffering in non-human animals}

Primates. Initial research with non-human subjects employed infant squirrel monkeys. A typical procedure involves separating the monkeys from their mother followed either by a reunion with her or placement in a familiar social environment, which results in a quick return to baseline cortisol levels (Hostinar et al., 2014). 
Coe et al. (1978) found that a previous relation with the buffer is a necessary condition for this effect to occur. These authors measured cortisol levels in mother and infant subjects across four conditions: (1) basal levels, (2) momentary separation followed by reunion of mother and infant, (3) infant removal from the group, and (4) mother removal from the group. They found that separation resulted in elevated cortisol levels in both mother and infant, although this was prevented if the separation was followed by immediate reunion. More importantly, this rise in cortisol levels in the infants was not prevented by the presence of another female adult, indicating that the relationship previously established with the mother plays a crucial role in the social buffering effect. A possible explanation for these results is that the mother's odor plays a role in buffering the stress response, similarly to what occurs in rodents.

However, a relationship with the mother may not be necessary for partners to become sources of social buffering. In this sense, Hennessy (1984) reared squirrel monkeys on inanimate maternal surrogates in individual cages. After one year, each subject was paired with a conspecific similarly raised for four weeks. The author found elevated cortisol levels when these monkeys were exposed to a novel environment alone, but not when they were tested in pairs. Nonetheless, the effect appears to be less reliable when a social partner different from the mother is involved. For example, Hennessy (1986) exposed adult female squirrel monkeys to a novel environment either alone or with a familiar partner. Depending on the condition, the partner was either familiar and judged to be affiliative with the subject, familiar and not judged to be affiliative, or unfamiliar. The author did not found a social buffering effect across any of the conditions. However, the methodology used for the formation of groups in this research seems to be confusing regarding the consideration of subjects as affiliative or non-affiliative.

Another procedure employed with squirrel monkeys consists of exposing the subjects to a predator before allowing them to be reunited with their partners. For example, Vogt, Coe, and Levine (1981) presented adult squirrel monkeys toa live boa constrictor snake, either in individual or group conditions. In the first condition, the subject was removed from the home cage and placed in a separate cage with the stimulus box on top. The group condition, on the other hand, involved placing the stimulus box on top of the home cage. In different conditions, the stimulus box could either be empty or contain the snake. Even though the authors did not find an elevation in cortisol levels produced by the presence of the snake, they did find such an elevation product of individual testing when compared with group testing,probably by mere exposure to the 
novel environment. These results suggest that presence of social partners (among other factors) may function as sources of social buffering.

In a follow-up study, Coe, Franklin, Smith, and Levine (1982) measured cortisol levels in males during four experimental conditions: (1) after pair formation, (2) after exposure to a novel environment, (3) after exposure to a predator, and (4) after ACTH administration. During conditions (2) and (3), animals were tested either alone or with their partner. Of interest here are the results of these two conditions. Interestingly, during condition (2), the authors found similar increments in cortisol levels when subjects were tested alone or in pairs, with half the subjects showing higher levels when tested with a partner. The same pattern was observed during condition (3). This led the authors to conclude that the presence of conspecifics alone may not be enough to produce social buffering, and that other factors may play a role (e.g., novelty of the setting in which the stressful situation occurs, number of conspecifics present and magnitude of the stressor).

Primate species other than squirrel monkey have also been studied. For example, Smith, McGreer-Whitworth, and French (1998) found that heterosexual partners serve as social buffers in adult marmosets. These authors isolated the subjects for two days either while accompanied by the partner or alone. Cortisol elevations were found only in the latter case. Furthermore, these elevations were not a consequence of partner-separation, since a second experiment showed that removing the partner from the home cage did not produce changes in cortisol levels. The authors concluded from this that the elevations were the result of novelty exposure, and that the partners were successful in buffering the stress response.

Stimuli other than the presence of a conspecific have also been studied in this species. Rukstalis and French (2005) performed an experiment in which adult marmosets were isolated from their pair mate and tested under either one of three conditions: (1) presentation of vocalizations from their mate, (2) presentation of vocalization from an unfamiliar conspecific of the opposite sex, or (3) absence of auditory stimuli. Cortisol levels were lower in subjects from condition (1) compared with subjects from conditions (2) or (3). The authors coined the term "vocal buffering" to refer to this particular example of social buffering.

Rodents. Social buffering research in rodents has revealed important age constraints as to which conspecifics can attenuate the stress response. For example, Hennessy et al., (2006) conducted an experiment in which male guinea pigs were exposed to a novel environment at four different stages of development (preweaning, 
periadolescent, sexually but not socially mature (i.e., they are not yet able to achieve the social status needed for reliable breeding success)and sexually and socially mature, and under three different conditions: (1) alone, (2) with an unfamiliar adult female, or (3) with the preferred adult female depending on the stage (based on behavioral observations). They found that, during the first stage, only the preferred female (the biological mother) functioned as a source of social buffering. The opposite result was found during periadolescence; the unfamiliar female, but not the preferred female, buffered the stress response. Neither the preferredor the unfamiliar female affected the stress response during the third stage, while the preferred female (but not the unfamiliar one) did buffer the response during the fourth stage.

Similarly, Hennessy, Maken, and Graves (2000) found that both the biological mother and an unfamiliar adult female reduced cortisol in periadolescent guinea pigs exposed to a novel environment for either 10, 30, 60, or 90 minutes. This was the case even when the interactions with both kinds of females were drastically different (e.g., the subjects exhibited more defensive behavior when in presence of the unfamiliar female). Interestingly, adult males appear to be unable to buffer the stress response in periadolescent and adult guinea pigs (Hennessy et al., 2002).

Age-dependency of the stimuli capable of buffering the stress response has also been observed in rats. Stanton and Levine (1990) performed three experiments in which the effectiveness of different stimuli was tested at different ages. In experiment 1 , an anesthetized dam was compared with an adult male rat at 12,16, and 20 days of age. A mild effectiveness of the male was found at 12 and 16 days (but not at 20), while the dam was found to inhibit the stress response at all three ages. Experiment 2 compared the anesthetized dam with an anesthetized sibling pup during the same three ages. While the dam was again found to inhibit stress responsiveness, the sibling resulted ineffective regardless of the age of the subjects. Experiment 3 examined the effectiveness of the dam during a period in which its biological and social significance is modified. A decremented effectiveness of the dam to buffer the stress response was found when subjects were tested on PND 20, 24 and 28.

This specificity of age to determine the ability of partners to buffer the stress response has also been observed in guinea pigs. Maken and Hennessy (2009) housed male subject with two females each, and tested them in a novel environment at PND 40, 120, 180, and 240. Each subject was evaluated under four different conditions: (1) alone, (2) in the presence of the preferred female (as determined from observations), (3) in the 
presence of the other female, and (4) with an unfamiliar female. No difference in social buffering was found among conditions (2), (3), and (4) at PND 40, 120, and 180, while only the preferred female was able to reduce the cortisol level at PND 240.

Similar to what occurs in other species, whether a prior relationship exists among subjects, seems to modulate the social buffering effect. In this sense, Armario, Luna, and Balasch (1983) found that adult rats exposed to a novel environment in the presence of a cage mate showed a higher corticosterone level when compared to rats that were exposed alone or in the presence of a rat from a different cage. No difference was found among the latter two groups. Similar results were observed by Armario, Ortiz, and Balasch (1983) when exposing rats to an open field while accompanied either by a familiar or an unfamiliar conspecific. Similarly, both the mother and unfamiliar adult females inhibit the stress response in pre and postweaning guinea pigs exposed to a novel environment, while littermates have no effect (Graves \& Hennessy, 2000). These results resemble those of Hennessy (1986) previously mentioned, in which familiar partners failed to buffer stress induced by novelty in adult squirrel monkeys.

Another factor that seems to modulate the effectiveness of a conspecific to buffer the stress response seems to be whether or not the potential buffer is stressed during the testing. Kiyokawa, Kikusui, Takeuchi, and Mori (2004) paired fear-conditioned rat subjects with either a fear-conditioned or a naive unfamiliar partner. The authors found that both kinds of partners buffered the stress response of the subjects during the test, but those that were not fear-conditioned were more effective. This suggests that stressed conspecifics are less effective in producing social buffering. A large body of literature which will be reviewed in the next section - shows that this is also the case when the buffer is the mother.

Again similar to primate subjects, the mother seems to be particularly effective in buffering the stress response of rodents at early stages. In the aforementioned study by Graves and Hennesy (2000), cortisol levels were lower when novelty exposure was carried out in the presence of the mother, compared to an unfamiliar female at the preweaning stage. This effect was not observed during the postweaning stage. Similarly, Hennessy and Ritchey (1987) found that cortisol elevation was attenuated when infant guinea pigs were exposed to a novel environment either with its conscious or anesthetized mother. This did not occur when the subjects were accompanied by an unfamiliar anesthetized lactating female. Social buffering by the mother is so strong that it works even when the stressor is a deadly threat (i.e., an adult male rat; Wiedenmayer et al., 
2003). These results are also in accordance with those previously mentioned about the age-dependence of subjects to benefit from the presence of different conspecifics. These results contrast sharply with those of Hennessy et al. (2000), in which periadolescent guinea pigs were equally buffered by their mother and by an unfamiliar female. This provides evidence to the hypothesis that the mother is particularly effective to buffer the stress response at early stages, but not later in life.

Interestingly, physical contact with the mother does not seem to be essential for this effect to occur. Hennessy (1988) exposed guinea pig pups to a novel environment in either one of three conditions: (1) alone, (2) in the presence of the mother separated by a wire-mesh partition, or (3) in the presence of the freely accessible mother. The author found a social buffering effect in both conditions (2) and (3). However, he also found that condition (3) was more effective than condition (2), indicating that physical contact with the mother is still more beneficial, albeit not essential. This result is similar to that of Rukstalis and French (2005) previously reviewed, in which vocalizations from the subjects' mates (but not from other conspecifics) produced social buffering in adult marmosets.

A different result was found in rats by Stanton et al. (1987). These authors carried out three experiments with 12,16 , and 20 day old rats. The stressor was exposure to a novel environment. In Experiment 1, the subjects were either allowed to suckle an anesthetized dam or received milk ingestion through an intraoral cannula. The first condition resulted in reduced corticosterone secretion at all ages, while the latter did not. In Experiment 2 subjects were allowed to contact to an anesthetized dam, with or without the opportunity to suckle. The results suggest thatit is the contact with the dam, and not the opportunity to suckle per se, what buffers the stress response. Both conditions were equally effective at all ages. Finally, Experiment 3 assessed whether physical contact is essential for this buffering to occur. Four conditions were compared: (1) pups that were allowed physical contact with a lactating female, (2) pups that were allowed physical contact with a virgin female, (3) pups that were presented with a lactating female (without physical contact allowed), and (4) pups that were presented with a virgin female (without physical contact allowed). Social buffering was only observed when physical contact was allowed, and contact with a virgin female was less effective at 20 days of age when compared to 12 and 16.

In summary, not only does the mother function as a potent stress buffer, but it also has been shown to regulate in HPA axis during development. Early experiments showed 
that rats are hyporesponsive to stress during PND 4-14. Thus, this period has been called the stress hyporesponsive period (SHRP; Levine, 2001; Moriceau\& Sullivan, 2004). Further research has shown that the HPA axis is in fact functional during this period, but that maternal stimuli suppress its activation. Specifically, pups in the SHRP can show corticosterone elevations if maternally deprived (Levine et al., 1991), but tactile stimulation from the mother maintains low corticosterone levels and prevents stressors from producing its release (Moriceau \& Sullivan, 2004).

\section{Inhibition of conditioned fear responses by social stimuli}

Almost all the studies reviewed so far have focused on regulation of the stress response produced by stimuli such as exposure to novel environments or predators. An important field of research comes from studies about whether the role of social stimuli in attenuation of the stress response, when the source of stress is a conditioned fear stimulus. An early study (Stanton et al., 1985) showed that partners can have inhibitory effects in the adrenocortical response induced by conditioned stimuli. Adult male squirrel monkeys were assigned to either one of two groups. In the first one, subjects were fear-conditioned with footshock as the unconditioned stimulus (US) and a flashing light as the conditioned stimulus (CS). Subjects from the second group received presentations of the CS without pairing. Afterwards, both groups were tested with the CS alone, either individually, in the presence of a partner or in the presence of the group. The authors found cortisol elevations only in subjects that received CS-US pairing, and only when they were tested individually; that is, conspecifics successfully attenuated the stress response evoked by the CS. However, no difference was found between animals tested in the presence of a single partner or in the presence of a larger group. Furthermore, social buffering facilitates the extinction of conditioned fear responses (Mikami et al., 2020).

Since then, a growing body of literature studying the regulation of conditioned fear responses by social stimuli has emerged. For example, Kiyokawa, Hiroshima, Takeuchi, and Mori (2014) exposed fear-conditioned and non-conditioned male rats to the corresponding CS (a 3-s, 8kHz, 80dB tone) either while alone or in the presence of a conspecific that was separated by a wire mesh barrier. The authors found corticosterone elevations only when the fear-conditioned subjects were tested alone. On the other hand, no difference was found among fear-conditioned subjects tested in the presence of a partner compared with non-conditioned subjects. However, this effect is absent when the subjects are separated from the partner by a transparent acrylic board, or when their main 
olfactory epithelium is lesioned (Kiyokawa et al., 2009). Together, these results imply that the olfactory system is essential for inhibition of the stress response to occur, while physical contact is not.

Visual stimuli produced bythe partner do not seem to be essential either. Takahashi et al. (2013) fear-conditioned male rats and then exposed them to the CS in a test box that was either clean or odorized by a partner that was previously kept there. The CS elicited fear conditioned responses in the first case, but failed to do so when the odor of the partner was presented. The effects of familiarity with the conspecific were analyzed in a subsequent study (Kiyokawa et al., 2014). The procedure was similar to the previous one, except that the odor could be either from a familiar conspecific or an unfamiliar one. Both odors were effective in suppressing the conditioned fear responses, but the odor from the familiar partner was more effective than that of the unfamiliar one. These results extend previous findings on partner familiarity to conditioned fear responses.

Experiments within this paradigm have also shed light on the frequent lack of correlation found between behavioral and physiological measures of stress (e.g., Coe et al., 1982; Vogt, et al., 1981). In this respect, Kiyokawa, Takeuchi, and Mori (2007) found differential effects of two experimental arrangements in male rats. In the first one, subjects were fear-conditioned to an auditory CS (with shock as the US) and, afterwards, pair-housed with an unfamiliar rat for 24 hours. This procedure resulted in a reduced autonomic response to the CS compared to control subjects, but had no effect on behavioral measures of stress. In the second arrangement, subjects were fear-conditioned in the same fashion as in the first one, and were then tested with the CS in the presence of an unfamiliar rat. The authors found that this condition had somewhat the opposite effect; behavioral signs of stress were reduced, but no difference was found in the autonomic response. Furthermore, the authors found that exposure in the presence of the unfamiliar rat following pair-housing reduced both behavioral and physiological signs of stress, showing that a combination of both arrangements may be the most effective way of attenuating the stress response.

All studies reviewed so far in this section have used male rats as subjects. This begs the question of whether female rats are also susceptible to this effect. In a study by Ishii, Kiyokawa, Takeuchi, and Mori (2016) the effect of social buffering in females was evaluated. In a first experiment, the authors found that the conditioned fear response did not depend on the stage of the estrus cycle. A second experiment showed that, when tested in presence of another female, the subject's response does not depend on the stage of the 
partner's estrus cycle either. Finally, Experiment 3 showed that HPA-axis activation was attenuated by the presence of another female when compared to alone testing, providing evidence of stress regulation of conditioned fear responses in female rats by social stimuli.

Usually, the goal of the experiments is to study how the buffer influences the responses of the experimental subjects. Recently, however, Kiyokawaand Takeuchi (2019) asked whether changes also occur in the buffer during exposure to the CS. The answer was positive: the conspecifics exhibited increased anogenital contact, allogrooming, and c-Fos expression in the paraventricular nucleus of the hypothalamus and central amígdala, indicating a state of distress. This demonstrates a mutual influence of the experimental subject and buffer during social buffering.

\section{Effects of rearing by a stressed mother}

Throughout the previous section numerous experiments showing how the presence of a conspecific can inhibit the adrenocortical response to stressors have been reviewed. Among them, several showed that the mother can be particularly effective in this regard, at least during early stages of development. Another common finding is that the effect depends on whether the buffer is under conditions of stress, in which case social buffering is not observed. Taking into account both of these findings, several authors have inquired about the effects of animals reared by a mother subjected to stressful conditions (for a review, see Walker et al., 2017).

Poor parenting conditions, such as insufficient access to resources by the mother, could function as a stressor for both the mother and the offspring (Perry et al., 2018). An animal model prominently used with this purpose consists of providing the mother with insufficient nesting material, which prevents the construction of an appropriate nest. This procedure has been dubbed the scarcity model and has proven to be stressful for the mother, as shown by increased basal corticosterone levels (Ivy et al., 2008). This, in turn, has a profound impact in maternal behavior. Specifically, behaviors that are considered to be beneficial (i.e., spending time in the nest with pups, keeping them together in the nest, nursing pups and grooming them) show a reduced frequency, while other behaviors considered to be detrimental (i.e., roughly transporting, stepping on pups, keeping them scattered across the home cage and self-grooming of the mother) become more frequent (Perry et al., 2018). Furthermore, corticosterone levels in the pups are increased as a result of this procedure, showing that rearing under these conditions is stressful for them (Rice et al., 2008). The usefulness of this paradigm thus becomes apparent, since stress 
experienced during the neonatal stage and infancy produces negative effects in the offspring that can be reflected even in later stages of development. Such effects include schizophrenia-like behavior (Girardi et al., 2014), mortality rate (McPherson et al., 2007), hyperglycemia (McPherson et al., 2009), hypercorticosteronemia and enhanced intestinal permeability (Moussaoui et al., 2017). An additional advantage of this model is that, in contrast with the experiments reviewed thus far, it can be used to study the effects of chronic stress.

The effects that the scarcity model produces on the pups has proven to be longlasting. For example, Guo, Wang, Mayer, and Holschneider (2015) found that rats reared under the scarcity condition exhibit visceral hyperalgesia during adulthood. This effect, however, appears to be exclusive to males (Prusator \& Greenwood- Van Meerveld, 2015). Similarly, rat pups reared with limited resources show disrupted social behavior during adolescence (Rincón-Cortés \& Sullivan, 2016). However, these effects also appear to be reversible, as shown by Maniam, Antoniadis, Le, and Morris (2016). These authors assigned rats to either scarcity or control conditions. At weaning, some of the subjects from the scarcity condition were switched to a diet high in fat and sugar, while the others were kept on a typical diet for laboratory rats. The authors found that subjects in the scarcity condition displayed more anxious-like behaviors when compared to the control group, but that this effect was reversed by the change in the diet.

This paradigm has been employed to model a wide variety of human phenomena. For example, Perry et al. (2018) used a scarcity model to study the effects of poverty on development. These authors assigned rodent mothers and their pups to either a scarcity rearing environment (with insufficient nesting material) or to a control condition (in which abundant nesting material was provided). Maternal behaviors that were categorized either as sensitive or negative were analyzed. Behaviors that promoted the survival of the pups were categorized as sensitive, and included the presence of the mother in the nest, nursing the pups and grooming them. Behaviors that put the pups at risk were categorized as negative and included, for example, rough transport of the pups and stepping on them. The goal was to assess whether low resources would affect caregiving quality and, in turn, if this would have a negative impact on pups' development. Furthermore, the ecological validity of the model was assessed by comparing the results with findings from a longitudinal study in humans under conditions of resource scarcity. Parental behaviors were similarly classified as either sensitive or negative. The results showed that rodent mothers in the scarcity condition displayed less sensitive and more negative behaviors 
towards the pups when compared to the control group. Also, pups reared under the scarcity condition showed decreased developmental competence when compared to subjects in the control condition (i.e., disrupted nipple attachment, distress vocalizations when in physical contact with an anesthetized mother, and reduced preference for maternal odor). Similar results were found on the human subjects, in which scarcity conditions positively correlated with decreased positive affect, decreased mental development, and decreased attention. From this, the authors conclude that altered caregiving quality may be one of the ways in which poverty impacts on development. However, they also note that the rodent model cannot fully capture the complexity of the human condition, and that findings from one model should be taken as complimentary of the other.

Since the scarcity procedure increases negative behaviors towards the pups, it has also been used as a model of maternal maltreatment (for a review, see Perry \& Sullivan, 2014). For example, Rincón-Cortés and Sullivan (2016) exposed rats to this procedure and then tested them for adult depressive-like behavior at three ages: (1) infancy, (2) periweaning and (3) adolescence. The results showed that the rough caregiving during infancy disrupted social behavior at periweaning and adolescence, although it had no effect on infant social behavior. These results are similar to those found with humans, in whom maltreatment and abuse during infancy result in social deficiencies, which in turn predict the development of psychopathology later in life (Alink et al., 2012; Lansford et al., 2002; Mason et al., 2004).

Interestingly, pups reared under conditions of rough caregiving still display attachment towards the mother. This persistence in approaching behavior appears to have evolved because parental care is essential for the survival of the pups (Sullivan, 2003). Even more interestingly, pups show preference for an odor paired with an abusive mother. For example, Roth and Sullivan (2005) assigned male and female rat pups to either one of four conditions: (1) paired condition, which consisted on pairing a novel odor (peppermint) with the abusive mother, (2) unpaired condition, presenting the odor 30 minutes before exposure to the abusive mother, (3) odor only condition, presenting the odor without exposure to maltreatment, and (4) maltreatment only condition, which consisted on exposing the pups to the abusive mother without presentations of the odor. The authors found that subjects in condition (1) showed a conditioned odor preference towards the peppermint, which was absent among subjects in conditions (2), (3) and (4). 
However, the fact that abused pups still develop attachment behaviors towards the mother does not imply that maltreatment goes without consequences. For example, Raineki, Cortés, Belnoue, and Sullivan (2012) found that abused pups exhibit deficits in social behavior at preweaning and adolescence, as measured by a diminished preference for a chamber where a conspecific is found. These authors also found depressive-like behavior during adolescence in rats that were abused as pups, as measured by an increased amount of time spent immobile during a forced swim test. Similar results were reported by Rincón-Cortés and Sullivan (2016), with depressive-like behavior observed during adulthood instead of adolescence. Paradoxically, these differences among experimental and control subjects are absent if the tests are carried out in the presence of the abusive mother's odor (Raineki et al., 2015). These results seem to indicate that pups can still benefit from an attachment relation with the mother, even under abusive rearing.

Another consequence of the scarcity model is that the mother's ability to buffer fear responses becomes compromised. Robinson-Drummer et al. (2019) exposed two groups of rats - one reared using the scarcity model and the other reared under normal conditions - to a fear-conditioning procedure either in the presence of their mother or alone. Conditioning took place during PND18 for some subjects and during PND28 for the rest. The CS was peppermint odor, which was unfamiliar to the subjects. At PND 18, the authors found evidence of social buffering in the subjects reared under normal conditions. Specifically, they found a lower freezing duration in subjects that were conditioned in the presence of the mother when compared to those conditioned alone. However, abusive rearing disrupted social buffering of pups by the mother at PN18. When tested at PND28, social buffering was observed in subjects reared under both conditions. Though, the effect was less robust in those subjects reared using the scarcity model. Yirmiya et al. (2020) found similar effects in humans. They compared the ability of the mother to buffer the stress responses in a group of adolescents that experienced early-life stress and with a group of adolescents raised under conditions of low stress. The authors only found evidence of social buffering in this latter group.

\section{The importance of odor for social buffering in rats}

This section will be focused exclusively in rat models. Specifically, the role of the olfactory system in the modulation of the social buffering effect will be discussed. In this regard, odor is important for two reasons; the first one is that olfactory stimuli from the buffer seem to be essential for social buffering to occur in this species (Kiyokawa et al., 
2009). The second is that, since rats are an altricial species, they strongly depend on odor to keep proximity to the dam early in life, which in turn is essential to ensure their survival (Sullivan, 2003). This is facilitated by an enhanced capacity to acquire odor preferences, along with a reduced capacity for the development of aversion to familiar odors. These particular learning capabilities are thought to have evolved in order to prevent pups from learning to avoid the mother, since caregiving often implies some painful stimulation. This enhanced/decreased capacity for learning odor preferences/avoidance lasts from PND 0 to 9 (for a thorough review, see Moriceau et al., 2010).

Works showing the importance of olfactory stimuli have already been reviewed in a previous section. For instance, Kiyokawa et al. (2009) showed that the presence of a partner suppresses conditioned fear responses, even if separated by a wire mesh. This effect is absent if they are separated by a transparent acrylic board instead, or if the subject's main olfactory epithelium is lesioned. Furthermore, Takahashi et al. (2013) found that conditioned fear responses are suppressed if the subjects are tested in a chamber previously odorized by a partner. Together, these results suggest that olfactory stimuli are both necessary and sufficient to produce social buffering of conditioned fear responses in rats.

The particular learning capabilities of newborn rats regarding odor preference have been repeatedly observed in classical conditioning experiments in which a painful unconditioned stimulus (shock) is paired with a neutral odor. The usual finding is that rats under nine days of age develop a preference for this odor, contrary to what happens during later stages of development, when aversion is developed instead (e.g., Camp \& Rudy, 1988). As mentioned in a previous section, an odor preference is also developed if the unconditioned stimulus is an abusive mother (Roth \& Sullivan, 2005). In view of this, the period ranging from PND 0 to 9 has been dubbed sensitive period (Moriceau et al., 2010). Interestingly, an odor paired with an electric shock of moderate intensity during this period will still be preferred if tested after PND 9, when avoidance is capable of being developed (Sullivan et al., 2000).

It is important to note that the inability to developed avoidance during the sensitive period is not due to an inability to feel pain. This is evidenced by the fact that unconditioned responses to shock during this period are not fundamentally different from those of adult rats (Sullivan et al., 2000). Instead, it appears that the inability to develop conditioned avoidance is due to the fact that the adrenocortical response during this period is suppressed by maternal stimuli. Indeed, Sullivan et al. (2000) showed that pups in the 
sensitive period are capable of developing odor avoidance if injected with corticosterone prior to training.

\section{Conclusions}

The social buffering effect is a complex phenomenon. Which social stimuli are capable of producing it depend critically on the developmental stage of the subject, with the mother tending to be effective during early stages and conspecifics during adolescence/adulthood. The mother is remarkably powerful in buffering the stress response during early stages of development, but even this robust effect can be modulated by the conditions of the buffer. Furthermore, the physiological state of the buffer also modulates the effect; specifically, no attenuation of the stress response occurs if the buffer is itself under a stressful state. For example, studies which employed scarcity models found that the scarcity conditions completely disrupt the ability of the mother to produce social buffering.

Table 1 summarizes the results reviewed in this paper. Interestingly, the results of research in both animal models and humans are congruent on two main ideas: 1 . The presence of a familiar conspecific decreases stress responses in threatening situations, 2. The effective buffer differs at each stage of ontogeny, suggesting that it is a dynamic process that depends on the maturation of the organisms.

Table 1.

Summary of the results reviewed in this paper.

\begin{tabular}{|c|c|c|c|c|}
\hline Reference & Species & Age & Design & Results \\
\hline $\begin{array}{l}\text { Hostinar et al. } \\
\qquad(2015)\end{array}$ & Human & $\begin{array}{l}\text { Infancy and } \\
\text { adolescence }\end{array}$ & $\begin{array}{l}\text { TSST in the } \\
\text { presence of } \\
\text { parents or } \\
\text { strangers }\end{array}$ & $\begin{array}{l}\text { Parents reduced } \\
\text { stress when } \\
\text { compared to } \\
\text { strangers in } \\
\text { infancy. No } \\
\text { difference in } \\
\text { adolescence }\end{array}$ \\
\hline $\begin{array}{l}\text { Seltzer et al. } \\
\qquad(2010)\end{array}$ & Human & Infancy & $\begin{array}{l}\text { Recovery of } \\
\text { TSST in the } \\
\text { presence of the } \\
\text { mother or an } \\
\text { experimenter } \\
\text { (with or without } \\
\text { the possibility of } \\
\text { speaking with the } \\
\text { mother) }\end{array}$ & $\begin{array}{l}\text { Presence of the } \\
\text { mother was more } \\
\text { effective than } \\
\text { speaking, which } \\
\text { was more } \\
\text { effective than the } \\
\text { experimenter } \\
\text { alone }\end{array}$ \\
\hline
\end{tabular}


Seltzer et

al.(2012)

Human

Infancy

Kirschbaum et al. (1995)

Human

Adulthood

Ditzen et al.

(2007)

Glynnet al.(1999)

Human

Heinrichs et al. (2003)

Human

Adulthood

Coe et al. (1978)

Squirrel monkey

Infancy

Vogt et al. (1981) Squirrel monkey

Coe et al. (1982) Squirrel monkey

Smith et al. (1998)
Marmoset
Adulthood

Adulthood

Adulthood

Adulthood
Similar to Seltzer,

Ziegler, and

Pollak (2010), with texting instead of speaking

No support, support from a stranger or support from the partner in the

TSST anticipation Women received either 10 minutes of physical contact with their partner, 10 minutes of verbal interaction, or no interaction during anticipation of TSST

Speech with male/female supportive/unsupp ortive feedback

TSST in presence/absence of best friend

Mother-infant dyads were separated with or without immediate reunion

Only the presence of the mother was effective

Only the partner was effective for men. Partner was less effective than strangers for women

Only physical contact was effective

Only positive feedback from female observer was effective

Presence of best friend was effective

Reunion with the mother (and not with another female) prevented increases in cortisol levels

Exposure to novel environment with unfamiliar, familiar affiliative, or familiar unaffiliative partner

Exposure to a predator alone or in group

Exposure to a novel environment/pred ator alone or with a partner

Isolation alone or with a partner
Neither condition was effective

Group-testing prevented increases in cortisol levels

Neither condition was effective

Partner prevented increases in cortisol levels 


\author{
Rukstalis\& \\ French (2005)
}

Hennessy et al., (2006)

Hennessy et al. (2000)

Stanton \& Levine (1990)

Maken\&

Hennessy (2009)

Armario et al.

(1983)

Graves

\&Hennessy(2000)

Kiyokawa et al (2004)
Guinea pig

Marmoset

Adulthood

Preweaning through adulthood

PND 12 through 24

Guinea pig

PND 40 through 240

Rat

Adulthood

Guinea pig

Rat

Postweaning
Isolation from their partner with exposure to vocalizations of the partner, an unfamiliar conspecific or neither

Exposure to a novel environment alone, with an unfamiliar adult female, with the preferred female at that age

Exposure to a novel

environment with the biological mother or an unfamiliar adult female

Comparison of the mother with other social stimuli at different ages

Exposure to a novel environment alone or with a preferred/unprefer red/unfamiliar female

Exposure to a novel environment alone, with a cage mate or a rat from a different cage

Exposure to a novel

environment with the mother, an unfamiliar female or a littermate Pairing of fearconditioned subjects with fearconditioned or naïve partner

\section{Only \\ vocalizations \\ from the partner were effective}

Preferred female was effective at preweaning and adulthood.

Unfamiliar female was effective at periadolescence.

\section{Both were effective}

The mother was effective from PND 12 through 20 , with a decreasing effect at PND 24 and 28. An adult male was effective at PND 12 and 16. A sibling pup was not effective at any age

All females were equally effective at PND 40, 120 and 180 . Only the preferred female was effective at

PND 240

Higher corticosterone levels in presence of the cage mate

Both the mother and the unfamiliar female are effective

Naïve partner was more effective 
Hennessy \&

Ritchey (1987)

Hennessy (1988)

Stanton et al. (1985)

Kiyokawa et al. (2014)

Takahashi et al. (2013)

Kiyokawa et al. (2014)

Ishii et al. (2016)

Perry et al. (2018)
Guinea pig

Infancy

Infancy

Guinea pig

Squirrel monkey

Adulthood

Adulthood

Rat

Adulthood

Adulthood

Rat

Rat

Adulthood

Infancy
Exposure to a

novel

environment with the mother or an unfamiliar female

Exposure to a novel

environment

alone or with the mother

(accessible or

inaccessible)

Fear-conditioned subjects were

tested alone, with a partner or with the group

Fear-conditioned subjects were tested alone or with a partner separated by a wire mesh barrier Fear-conditioned subjects were tested in a box either clean or odorized by a partner

Similar to

Takahashi et al. (2013), with odor from either a familiar or an unfamiliar partner

Studied social buffering on female subjects in different stages of the estrus cycle of the subjects and the partners

Rearing with abundant/scarce bedding material
Only the mother is effective

The mother was more effective when accessible

Testing with a partner or the group were equally effective

The partner was effective

The odor was effective

Both odors were effective, with a stronger effect by the familiar partner's odor

The stage of the estrus cycle in either the subject or the partner had no effect. The partner was effective

Mothers in the scarcity condition displayed less sensitive and more negative towards the pups. Pups in the scarcity condition showed decreased developmental competence 
Rincón-Cortés \& Sullivan (2016)

Roth \& Sullivan (2005)

Robinson-

Drummer et al. (2019)
Rat

Infancy through adolescence

Infancy

Rat

Subjects exposed to the scarcity procedure were tested for depressive-like behaviors

A novel odor was paired with the abusive mother, with unpaired controls

Subjects reared under scarce or normal conditions were fearconditioned in presence or absence of the mother, at PND 18 or 28
Disrupted social behavior at periweaning and adolescence

Pairings resulted in an odor preference

Social buffering at PND 18 in subjects reared under normal conditions, especially if conditioned in presence of the mother, with no effect in scarcereared subjects.

At PND 28, social buffering was found in all groups, being stronger in normal-reared pups 


\section{References}

Abelson, J. L., Erickson, T. M., Mayer, S. E., Crocker, J., Briggs, H., Lopez-Duran, N. L., \&Liberzon, I. (2014). Brief cognitive intervention can modulate neuroendocrine stress responses to the Trier Social Stress Test: Buffering effects of a compassionate goal orientation. Psychoneuroendocrinology, 44, 60-70. https://doi.org/10.1016/j.psyneuen.2014.02.016

Adams, R. E., Santos, J. B., \& Bukowski, W. M. (2011). The presence of a best friend buffers the effects of negative experiences. Developmental Psychology, 47, 1786-1791. https://doi.org/10.1037/a0025401

Albers, E. M., Riksen-Walraven, J. M., Sweep, F. C. G. J., \& de Weerth, C. (2008). Maternal behavior predicts infant cortisol recovery from a mild everyday stressor. Journal of Child Psychology and Psychiatry, 49, 97-103. https://doi.org/10.1111/j.1469-7610.2007.01818.x

Alink, L. R., Cicchetti, D., Kim, J., \&Rogosch, F. A. (2012). Longitudinal associations among child maltreatment, social functioning, and cortisol regulation. Developmental psychology, 48(1), 224-236. https://doi.org/10.1037/a0024892

Armario, A., Luna, G., \&Balasch, J. (1983). The effect of conspecifics on corticoadrenal response of rats to a novel environment. Behavioral and neural biology, 37(2), 332-337. https://doi.org/10.1016/S01631047(83)91425-5

Armario, A., Ortiz, R., \&Balasch, J. (1983). Corticoadrenal and behavioral response to open field in pairs of male rats either familiar or non-familiar to each other. Experientia, 39(11), 1316-1317. https://doi.org/10.1007/BF01990391

Biggs, A., Brough, P., \& Drummond, S. (2017). Lazarus and Folkman's psychological stress and coping theory. In The handbook of stress and health: A guide to research and practice (351-364). APA.

Camp, L. L., \& Rudy, J. W. (1988). Changes in the categorization of appetitive and aversive events during postnatal development of the rat. Developmental Psychobiology: The Journal of the International Society for Developmental Psychobiology, 21(1), 25-42. https://doi.org/10.1002/dev.420210103

Childs, E., Vicini, L. M., \& De Wit, H. (2006). Responses to the Trier Social Stress Test (TSST) in single versus grouped participants. Psychophysiology, 43(4), 366-371. https://doi.org/10.1111/j.1469-8986.2006.00414.x

Cobb, S. (1976). Social support as a moderator of life stress. Psychosomatic medicine, 38(5), 300-314. https://doi.org/10.1097/00006842-197609000$\underline{00003}$

Coe, C. L., Franklin, D., Smith, E. R., \& Levine, S. (1982). Hormonal responses accompanying fear and agitation in the squirrel monkey. Physiology \& behavior, 29(6), 1051-1057. https://doi.org/10.1016/0031-9384(82)90297-9

Coe, C. L., Mendoza, S. P., Smotherman, W. P., \& Levine, S. (1978). Mother-infant attachment in the squirrel monkey: Adrenal response to separation. Behavioral Biology, 22, 256-263. https://doi.org/10.1016/S00916773(78)92305-2

Cohen, B. E., Edmondson, D., \&Kronish, I. M. (2015). State of the art review: depression, stress, anxiety, and cardiovascular disease. American journal of hypertension, 28(11), 1295-1302. https://doi.org/10.1093/ajh/hpv047 
Cohen, S., \& McKay, G. (1984). Social support, stress and the buffering hypothesis: A theoretical analysis. In A. Baum, S.E. Taylor, \& J.E. Singer (Eds.) Handbook of psychology and health, Vol. 4 (pp. 253-267). Hillsdale.

Cohen, S., \& Wills, T. A. (1985). Stress, social support, and the buffering hypothesis. Psychological bulletin, 98(2), 310-357. https://doi.org/10.1037/0033-2909.98.2.310

Cooper, C., \&Dewe, P. J. (2008). Stress: A brief history. John Wiley\&Sons.

Culbert, B. M., Gilmour, K. M., \&Balshine, S. (2019). Social buffering of stress in a group-living fish. Proceedings of the Royal Society B, 286(1910), 20191626.

Dean, A., \& Lin, N. (1977). The stress-buffering role of social support. Journal of Nervous and Mental Disease, 165(6), 403-417. https://doi.org/10.1097/00005053-197712000-00006

Dinan, T. G. (1994). Glucocorticoids and the genesis of depressive illness a psychobiological model. The British Journal of Psychiatry, 164(3), 365-371. https://doi.org/10.1192/bjp.164.3.365

Ditzen, B., Neumann, I. D., Bodenmann, G., von Dawans, B., Turner, R. A., Ehlert, U., \&Heinrichs, M. (2007). Effects of different kinds of couple interaction on cortisol and heart rate responses to stress in women.

Psychoneuroendocrinology, 32(5), 565-574. https://doi.org/10.1016/j.psyneuen.2007.03.011

Duval, F., González, F., \& Rabia, H. (2010). Neurobiología del estrés. Revista Chilena de Neuro-Psiquiatría, 48(4), 307-318. https://doi.org/10.4067/s071792272010000500006

Eisenberger, N. I., Taylor, S. E., Gable, S. L., Hilmert, C. J., \& Lieberman, M. D. (2007). Neural pathways link social support to attenuated neuroendocrine stress responses. Neuroimage, 35(4), 1601-1612. https://doi.org/10.1016/j.neuroimage.2007.01.038

Girardi, C. E. N., Zanta, N. C., \&Suchecki, D. (2014). Neonatal stress-induced affective changes in adolescent Wistar rats: early signs of schizophrenia-like behavior. Frontiers in behavioral neuroscience, 8, 319.

https://doi.org/10.3389/fnbeh.2014.00319

Glynn, L. M., Christenfeld, N., \&Gerin, W. (1999). Gender, social support, and cardiovascular responses to stress. Psychosomatic medicine, 61(2), 234-242. https://doi.org/10.1097/00006842-199903000-00016

Gold, P. W., Machado-Vieira, R., \&Pavlatou, M. G. (2015). Clinical and biochemical manifestations of depression: relation to the neurobiology of stress. Neural plasticity, 2015, 1-11. https://doi.org/10.1155/2015/581976

Graves, F. C., \& Hennessy, M. B. (2000). Comparison of the effects of the mother and an unfamiliar adult female on cortisol and behavioral responses of preand postweaning guinea pigs. Developmental Psychobiology: The Journal of the International Society for Developmental Psychobiology, 36(2), 91-100. https://doi.org/10.1002/(SICI)1098-2302(200003)36:2<91::AIDDEV1>3.0.CO;2-1

Grossman, P., Niemann, L., Schmidt, S., \& Walach, H. (2004). Mindfulness-based stress reduction and health benefits: A meta-analysis. Journal of psychosomatic research, 57(1), 35-43. https://doi.org/10.1016/S00223999(03)00573-7

Gunnar, M. R., \& Hostinar, C. E. (2015). The social buffering of the hypothalamicpituitary-adrenocortical axis in humans: Developmental and experiential 
determinants. Social neuroscience, 10(5), 479-488. https://doi.org/10.1080/17470919.2015.1070747

Guo, Y., Wang, Z., Mayer, E. A., \&Holschneider, D. P. (2015). Neonatal stress from limited bedding elicits visceral hyperalgesia in adult rats. Neuroreport, 26(1), 13-16. https://doi.org/10.1097/WNR.0000000000000292

Hanson, J. D., Larson, M. E., \&Snowdon, C. T. (1976). The effects of control over high intensity noise on plasma cortisol levels in rhesus monkeys. Behavioral Biology, 16(3), 333-340. https://doi.org/10.1016/S0091-6773(76)91460-7

Heinrichs, M., Baumgartner, T., Kirschbaum, C., \&Ehlert, U. (2003). Social support and oxytocin interact to suppress cortisol and subjective responses to psychosocial stress. Biological Psychiatry, 54, 1389-1398. https://doi.org/10.1016/S0006-3223(03)00465-7

Hennessy, M. B. (1984). Presence of companion moderates arousal of monkeys with restricted social experience. Physiology \& Behavior, 33, 693-698. https://doi.org/10.1016/0031-9384(84)90033-7

Hennessy, M. B. (1986). Effects of social partners on pituitary-adrenal activity during novelty exposure in adult female squirrel monkeys. Physiology \& behavior, 38(6), 803-807. https://doi.org/10.1016/0031-9384(86)90046-6

Hennessy, M. B. (1988). Both prevention of physical contact and removal of distal cues mediate cortisol and vocalization responses of guinea pig pups to maternal separation in a novel environment. Physiology \& behavior, 43(6), 729-733. https://doi.org/10.1016/0031-9384(88)90369-1

Hennessy, M. B., Hornschuh, G., Kaiser, S., \&Sachser, N. (2006). Cortisol responses and social buffering: a study throughout the life span. Hormones and Behavior, 49(3), 383-390. https://doi.org/10.1016/j.yhbeh.2005.08.006

Hennessy, M. B., Kaiser, S., \&Sachser, N. (2009). Social buffering of the stress response: diversity, mechanisms, and functions. Frontiers in neuroendocrinology, 30(4), 470-482. https://doi.org/10.1016/j.yfrne.2009.06.001

Hennessy, M. B., Maken, D. S., \& Graves, F. C. (2000). Consequences of the presence of the mother or unfamiliar adult female on cortisol, ACTH, testosterone and behavioral responses of periadolescent guinea pigs during exposure to novelty. Psychoneuroendocrinology, 25(6), 619-632. https://doi.org/10.1016/S0306-4530(00)00014-7

Hennessy, M. B., O'Leary, S. K., Hawke, J. L., \& Wilson, S. E. (2002). Social influences on cortisol and behavioral responses of preweaning, periadolescent, and adult guinea pigs. Physiology \& Behavior, 76(2), 305314. https://doi.org/10.1016/S0031-9384(02)00712-6

Hennessy, M. B., \& Ritchey, R. L. (1987). Hormonal and behavioral attachment responses in infant guinea pigs. Developmental Psychobiology: The Journal of the International Society for Developmental Psychobiology, 20(6), 613625. https://doi.org/10.1002/dev.420200607

Herman, J. P., \&Cullinan, W. E. (1997). Neurocircuitry of stress: central control of the hypothalamo-pituitary-adrenocortical axis. Trends in neurosciences, 20(2), 78-84. https://doi.org/10.1016/S0166-2236(96)10069-2

Hostinar, C. E., Johnson, A. E., \& Gunnar, M. R. (2015). Parent support is less effective in buffering cortisol stress reactivity for adolescents compared to children. Developmental Science, 18, 281-297. https://doi.org/10.1111/desc. 12195 
Hostinar, C. E., Sullivan, R. M., \& Gunnar, M. R. (2014). Psychobiological mechanisms underlying the social buffering of the hypothalamic-pituitaryadrenocortical axis: A review of animal models and human studies across development. Psychological bulletin, 140(1), 256-282. https://doi.org/10.1037/a0032671

Ishii, A., Kiyokawa, Y., Takeuchi, Y., \& Mori, Y. (2016). Social buffering ameliorates conditioned fear responses in female rats. Hormones and behavior, 81, 53-58. https://doi.org/10.1016/j.yhbeh.2016.03.003

Ivy, A. S., Brunson, K. L., Sandman, C., \& Baram, T. Z. (2008). Dysfunctional nurturing behavior in rat dams with limited access to nesting material: a clinically relevant model for early-life stress. Neuroscience, 154(3), 11321142. https://doi.org/10.1016/j.neuroscience.2008.04.019

Kelly, M. M., Tyrka, A. R., Anderson, G. M., Price, L. H., \& Carpenter, L. L. (2008). Sex differences in emotional and physiological responses to the Trier Social Stress Test. Journal of behavior therapy and experimental psychiatry, 39(1), 87-98. https://doi.org/10.1016/j.jbtep.2007.02.003

Kirschbaum, C., Klauer, T., Filipp, S. H., \& Hellhammer, D. H. (1995). Sex-specific effects of social support on cortisol and subjective responses to acute psychological stress. Psychosomatic medicine, 57(1), 23-31. https://doi.org/10.1097/00006842-199501000-00004

Kirschbaum, C., Pirke, K.-M., \&Hellhammer, D. H. (1993). The "Trier Social Stress Test" - A tool for investigating psychobiological stress responses in a laboratory setting.Neuropsychobiology, 28(1-2), 76-81. https://doi.org/10.1159/000119004

Kiyokawa, Y., Hiroshima, S., Takeuchi, Y., \& Mori, Y. (2014). Social buffering reduces male rats' behavioral and corticosterone responses to a conditioned stimulus. Hormones and behavior, 65(2), 114-118. https://doi.org/10.1016/j.yhbeh.2013.12.005

Kiyokawa, Y., Honda, A., Takeuchi, Y., \& Mori, Y. (2014). A familiar conspecific is more effective than an unfamiliar conspecific for social buffering of conditioned fear responses in male rats. Behavioural brain research, 267, 189-193. https://doi.org/10.1016/j.bbr.2014.03.043

Kiyokawa, Y., Kikusui, T., Takeuchi, Y., \& Mori, Y. (2004). Partner's stress status influences social buffering effects in rats. Behavioral Neuroscience, 118, 798-804. https://doi.org/10.1037/0735-7044.118.4.798

Kiyokawa, Y., Li, Y., \&Takeuchi, Y. (2019). A dyad shows mutual changesduring social buffering of conditionedfear responses in malerats. Behaviouralbrainresearch, 366, 45-55.

Kiyokawa, Y., Takeuchi, Y., Nishihara, M., \& Mori, Y. (2009). Main olfactory system mediates social buffering of conditioned fear responses in male rats. European Journal of Neuroscience, 29(4), 777-785. https://doi.org/10.1111/j.1460-9568.2009.06618.x

Lansford, J. E., Dodge, K. A., Pettit, G. S., Bates, J. E., Crozier, J., \&Kaplow, J. (2002). A 12-year prospective study of the long-term effects of early child physical maltreatment on psychological, behavioral, and academic problems in adolescence. Archives of pediatrics \& adolescent medicine, 156(8), 824830. https://doi.org/10.1001/archpedi.156.8.824

LeResche, L., \& Dworkin, S. F. (2002). The role of stress in inflammatory disease, including periodontal disease: review of concepts and current findings. 
Periodontology 2000, 30(1), 91-103. https://doi.org/10.1034/j.16000757.2002.03009.x

Levine, S. (2001). Primary social relationships influence the development of the hypothalamic-pituitary-adrenal axis in the rat. Physiology \& behavior, 73(3), 255-260. https://doi.org/10.1016/S0031-9384(01)00496-6

Levine, S., Huchton, D. M., Wiener, S. G., \& Rosenfeld, P. (1991). Time course of the effect of maternal deprivation on the hypothalamic- pituitary- adrenal axis in the infant rat. Developmental Psychobiology: The Journal of the International Society for Developmental Psychobiology, 24(8), 547-558. https://doi.org/10.1002/dev.420240803

Levine, S., and Ursin, H. (1991). What is stress?. In M. R. Brown. G. F. Koob, and C. Rivier (Eds.), Stress, Neurobiology and Neuroendocrinology (pp. 3-21). Dekker.

Maken, D. S., \& Hennessy, M. B. (2009). Development of selective social buffering of the plasma cortisol response in laboratory-reared male guinea pigs (Caviaporcellus). Behavioral Neuroscience, 123, 347-355. https://doi.org/10.1037/a0015034

Maniam, J., Antoniadis, C. P., Le, V., \& Morris, M. J. (2016). A diet high in fat and sugar reverses anxiety-like behaviour induced by limited nesting in male rats: impacts on hippocampal markers. Psychoneuroendocrinology, 68, 202-209. https://doi.org/10.1016/j.psyneuen.2016.03.007

Mason, W. A., Kosterman, R., Hawkins, J. D., Herrenkohl, T. I., Lengua, L. J., \& McCauley, E. (2004). Predicting depression, social phobia, and violence in early adulthood from childhood behavior problems. Journal of the American Academy of Child \& Adolescent Psychiatry, 43(3), 307-315. https://doi.org/10.1097/00004583-200403000-00012

McEwen, B. S., \&Sapolsky, R. M. (1995). Stress and cognitive function. Current opinion in neurobiology, 5(2), 205-216. https://doi.org/10.1016/09594388(95)80028-X

McPherson, R. J., Gleason, C., Mascher-Denen, M., Chan, M., Kellert, B., \&Juul, S. E. (2007). A new model of neonatal stress which produces lasting neurobehavioral effects in adult rats. Neonatology, 92(1), 33-41. https://doi.org/10.1159/000100084

McPherson, R. J., Mascher-Denen, M., \&Juul, S. E. (2009). Postnatal stress produces hyperglycemia in adult rats exposed to hypoxia-ischemia. Pediatric research, 66(3), 278-282. https://doi.org/10.1203/PDR.0b013e3181b1bd1b

Mikami, K., Kiyokawa, Y., Ishii, A., \&Takeuchi, Y. (2020). Social bufferingenhancesextinction of conditionedfear responses byreducingcorticosteronelevels in malerats. Hormones and behavior, 118, 104654.

Moriceau, S., Roth, T. L., \& Sullivan, R. M. (2010). Rodent model of infant attachment learning and stress. Developmental psychobiology, 52(7), 651660. https://doi.org/10.1002/dev.20482

Moriceau, S., \& Sullivan, R. M. (2004). Corticosterone Influences on Mammalian Neonatal Sensitive-Period Learning. Behavioral Neuroscience, 118(2), 274281. https://doi.org/10.1037/0735-7044.118.2.274

Moussaoui, N., Jacobs, J. P., Larauche, M., Biraud, M., Million, M., Mayer, E., \&Taché, Y. (2017). Chronic early-life stress in rat pups alters basal corticosterone, intestinal permeability, and fecal microbiota at weaning: 
influence of sex. Journal of neurogastroenterology and motility, 23(1), 135143. https://doi.org/10.5056/jnm16105

Nachmias, M., Gunnar, M., Mangelsdorf, S., Parritz, R. H., \& Buss, K. (1996). Behavioral inhibition and stress reactivity: The moderating role of attachment security. Child development, 67(2), 508-522. https://doi.org/10.1111/j.14678624.1996.tb01748.x

Pechtel, P., \&Pizzagalli, D. A. (2011). Effects of early life stress on cognitive and affective function: an integrated review of human literature.

Psychopharmacology, 214(1), 55-70. https://doi.org/10.1007/s00213-0102009-2

Perry, R. E., Finegood, E. D., Braren, S. H., Dejoseph, M. L., Putrino, D. F., Wilson, D. A., ... \& Family Life Project Key Investigators. (2018). Developing a neurobehavioral animal model of poverty: Drawing cross-species connections between environments of scarcity-adversity, parenting quality, and infant outcome. Development and psychopathology, 1-20. https://doi.org/10.1017/S095457941800007X

Perry, R., \& Sullivan, R. M. (2014). Neurobiology of attachment to an abusive caregiver: Short- term benefits and long- term costs. Developmental psychobiology, 56(8), 1626-1634. https://doi.org/10.1002/dev.21219

Prusator, D. K., \& Greenwood- Van Meerveld, B. (2015). Gender specific effects of neonatal limited nesting on viscerosomatic sensitivity and anxiety- like behavior in adult rats. Neurogastroenterology\& Motility, 27(1), 72-81. https://doi.org/10.1111/nmo.12472

Raineki, C., Cortés, M. R., Belnoue, L., \& Sullivan, R. M. (2012). Effects of earlylife abuse differ across development: Infant social behavior deficits are followed by adolescent depressive-like behaviors mediated by the amygdala. Journal of Neuroscience, 32(22), 7758-7765. https://doi.org/10.1523/JNEUROSCI.5843-11.2012

Raineki, C., Sarro, E., Rincón-Cortés, M., Perry, R., Boggs, J., Holman, C. J., ... \& Sullivan, R. M. (2015). Paradoxical neurobehavioral rescue by memories of early-life abuse: The safety signal value of odors learned during abusive attachment. Neuropsychopharmacology, 40(4), 906-914. https://doi.org/10.1038/npp.2014.266

Ricci-Bonot, C., Romero, T., Nicol, C., \& Mills, D. (2021). Social buffering in horses (Equuscaballus): Influence of context, companionfamiliarity and companion habituation. Research Square, [preprint] https://doi.org/10.21203/rs.3.rs-266932/v1

Rice, C. J., Sandman, C. A., Lenjavi, M. R., \& Baram, T. Z. (2008). A novel mouse model for acute and long-lasting consequences of early life stress. Endocrinology, 149(10), 4892-4900. https://doi.org/10.1210/en.2008-0633

Rincón-Cortés, M., \& Sullivan, R. M. (2016). Emergence of social behavior deficit, blunted corticolimbic activity and adult depression-like behavior in a rodent model of maternal maltreatment. Translational psychiatry, 6(10), e930. https://doi.org/10.1038/tp.2016.205

Redinbaugh, E. M., MacCallum, R. C., \&Kiecolt-Glaser, J. K. (1995). Recurrent syndromal depression in caregivers. Psychology and aging, 10(3), 358-368. https://doi.org/10.1037/0882-7974.10.3.358

Robinson-Drummer P.A., Opendak M., Blomkvist A., Chan S., Tan S., Delmer C., Wood K., Sloan A., Jacobs L., Fine E., Chopra D., Sandler C., Kamenetzky G., \& Sullivan R.M. (2019) Infant trauma alters social buffering of threat 
learning: emerging role of prefrontal cortex in preadolescence. Front. Behav. Neurosci. 13:132. https://doi.org/10.3389/fnbeh.2019.00132

Roth, T. L., \& Sullivan, R. M. (2005). Memory of early maltreatment: neonatal behavioral and neural correlates of maternal maltreatment within the context of classical conditioning. Biological psychiatry, 57(8), 823-831. https://doi.org/10.1016/j.biopsych.2005.01.032

Rukstalis, M., \& French, J. A. (2005). Vocal buffering of the stress response: exposure to conspecific vocalizations moderates urinary cortisol excretion in isolated marmosets. Hormones and behavior, 47(1), 1-7. https://doi.org/10.1016/j.yhbeh.2004.09.004

Seltzer, L. J., Prososki, A. R., Ziegler, T. E., \&Pollak, S. D. (2012). Instant messages vs. speech: Hormones and why we still need to hear each other. Evolution and Human Behavior, 33, 42-45. https://doi.org/10.1016/j. evolhumbehav.2011.05.004

Seltzer, L. J., Ziegler, T. E., \&Pollak, S. D. (2010). Social vocalizations can release oxytocin in humans. Proceedings of the Royal Society B: Biological Sciences, 277, 2661-2666. https://doi.org/10.1098/rspb.2010.0567

Smith, T. E., McGreer-Whitworth, B., \& French, J. A. (1998). Close proximity of the heterosexual partner reduces the physiological and behavioral consequences of novel-cage housing in black tufted-ear marmosets (Callithrixkuhli).

Hormones and Behavior, 34(3), 211-222. https://doi.org/10.1006/hbeh.1998.1469

Stanton, M. E., Patterson, J. M., \& Levine, S. (1985). Social influences on conditioned cortisol secretion in the squirrel monkey. Psychoneuroendocrinology, 10(2), 125-134. https://doi.org/10.1016/03064530(85)90050-2

Stanton, M. E., Wallstrom, J., \& Levine, S. (1987). Maternal contact inhibits pituitary- adrenal stress responses in preweanling rats. Developmental Psychobiology: The Journal of the International Society for Developmental Psychobiology, 20(2), 131-145. https://doi.org/10.1002/dev.420200204

Stanton, M. E., \&Levine, S. (1990). Inhibition of infant glucocorticoid stress response: specific role of maternal cues. Developmental Psychobiology: The Journal of the International Society for Developmental Psychobiology, 23(5), 411-426. https://doi.org/10.1002/dev.420230504

Sullivan, R. M. (2003). Developing a sense of safety. Annals of the New York Academy of Sciences, 1008(1), 122-131. https://doi.org/10.1196/annals.1301.013

Sullivan, R. M., Landers, M., Yeaman, B., \& Wilson, D. A. (2000). Neurophysiology: Good memories of bad events in infancy. Nature, 407(6800), 38-39. https://doi.org/10.1038/35024156

Takahashi, Y., Kiyokawa, Y., Kodama, Y., Arata, S., Takeuchi, Y., \& Mori, Y. (2013). Olfactory signals mediate social buffering of conditioned fear responses in male rats. Behavioural brain research, 240, 46-51. https://doi.org/10.1016/j.bbr.2012.11.017

Taylor, S. E., Burklund, L. J., Eisenberger, N. I., Lehman, B. J., Hilmert, C. J., \& Lieberman, M. D. (2008). Neural bases of moderation of cortisol stress responses by psychosocial resources. Journal of Personality and Social Psychology, 95, 197-211. https://doi.org/10.1037/0022-3514.95.1.197 
Veenit, V., Riccio, O., \& Sandi, C. (2014). CRHR1 links peripuberty stress with deficits in social and stress-coping behaviors. Journal of psychiatric research, 53, 1-7. https://doi.org/10.1016/j.jpsychires.2014.02.015

Vogt, J. L., Coe, C. L., \& Levine, S. (1981). Behavioral and adrenocorticoid responsiveness of squirrel monkeys to a live snake: is flight necessarily stressful?Behavioral and Neural Biology, 32(4), 391-405. https://doi.org/10.1016/s0163-1047(81)90826-8

Walker, C. D., Bath, K. G., Joels, M., Korosi, A., Larauche, M., Lucassen, P. J., ... \&Taché, Y. (2017). Chronic early life stress induced by limited bedding and nesting (LBN) material in rodents: critical considerations of methodology, outcomes and translational potential. Stress, 20(5), 421-448. https://doi.org/10.1080/10253890.2017.1343296

Watts, A. G. (2007). Anatomy of the HPA axis. Stress science: Neuroendocrinology, 201-203.

Weiner, H. (1991). Behavioral biology of stress and psychosomatic medicine. In M. R. Brown. G. F. Koob, and C. Rivier (Eds.), Stress, Neurobiology and Neuroendocrinology (pp. 23-51). Dekker.

Wiedenmayer, C. P., Magarinos, A. M., McEwen, B. S., \& Barr, G. A. (2003). Mother lowers glucocorticoid levels of preweaning rats after acute threat. Annals of the New York Academy of Sciences, 1008(1), 304-307. https://doi.org/10.1196/annals.1301.038

Yada, T., \&Tort, L. (2016). Stress and diseaseresistance: immunesystem and immunoendocrineinteractions. In Fishphysiology (Vol. 35, pp. 365-403). AcademicPress.

Yirmiya, K., Motsan, S., Zagoory-Sharon, O., \&Feldman, R. (2020). Human attachmenttriggersdifferent social bufferingmechanismsunderhigh and lowearlylife stress rearing. International Journal of Psychophysiology, 152, $72-80$ 